\title{
Gerenciamento da dor na Síndrome Pós-poliomielite: estudo de caso
}

\section{Pain management in Postpoliomyelitis Syndrome: case study}

Marco Orsini ${ }^{1}$, Nelson Kale Júnior ${ }^{2}$, Mariana Pimentel Mello ${ }^{3}$, Dionis Machado ${ }^{4}$, Victor Hugo Bastos 5 , Daniel Joppert ${ }^{6}$, Carlos Henrique Melo Reis ${ }^{7}$, Júlio Guilherme Silva ${ }^{8}$, Acary Bulle Oliveira ${ }^{9}$, Sara Lúcia de Menezes $^{10}$, Marcos Raimundo de Freitas ${ }^{11}$

Estudo desenvolvido no Serviço de Neurologia da Universidade Federal Fluminense (UFF) - Niterói (RJ), Brasil.

${ }^{1}$ Doutor em Neurologia; Professor Colaborador do Serviço de Neurologia e do Programa de Mestrado em Ciências da Reabilitação do Centro Universitário Augusto Motta (UNISUAM); Interno de Medicina pela Universidade do Grande Rio (UNIRIO) - Duque de Caxias (RJ), Brasil.

2 Especialista em Fisioterapia Neurológica pela Escola Superior de Ensino Helena Antipoff (ESEHA) - Niterói (RJ), Brasil.

${ }^{3}$ Fisioterapeuta do Programa de Iniciação Cientifica da UFF - Niterói (RJ), Brasil.

${ }^{4}$ Fisioterapeuta; Mestre e Doutorando em Mapeamento Cerebral pela Universidade Federal do Rio de Janeiro (UFRJ) - Rio de Janeiro (RJ), Brasil.

${ }^{5}$ Fisioterapeuta; Doutor em Mapeamento Cerebral pela UFRJ Rio de Janeiro (RJ), Brasil.

${ }^{6}$ Fisioterapia; Especialista em Fisioterapia Neurológica pela ESEHA - Niterói (RJ), Brasil.

7 Neurologista; Mestre em Neurologia pela UFRJ - Rio de Janeiro (RJ), Brasil.

${ }^{8}$ Fisioterapeuta; Doutor em Mapeamento Cerebral pela UFRJ; Professor do Programa de Mestrado em Ciências da Reabilitação do UNISUAM - Rio de Janeiro (RJ), Brasil.

9 Neurologista; Doutor em Neurologia pela Escola Paulista de Medicina (UNIFESP) - São Paulo (SP), Brasil.

${ }^{10}$ Fisioterapeuta; Doutora em Fisiologia pela UFRJ; Professora do Programa de Mestrado em Ciências da Reabilitação do UNISUAM - Rio de Janeiro (RJ), Brasil. ${ }^{11}$ Neurologista; Professor Titular e Chefe do Serviço de Neurologia da UFF Niterói (RJ), Brasil.

ENDEREÇO PARA CORRESPONDÊNCIA Prof. Marco Orsini - Rua Herotides de Oliveira, 2 - compl. 801 - Jardim Icaraí - CEP: 24230-230 - Niterói (RJ), Brasil E-mail: orsinimarco@hotmail.com

APRESENTAÇ̃̃O set. 2010

ACEITO PARA PUBLICAÇ̃̃O abr. 2011

FONTE DE FINANCIAMENTO Programa de Mestrado Acadêmico em Ciências da Reabilitação - Centro Universitário Augusto Motta (UNISUAM)

CONFLITO DE INTERESSE

nada a declarar
RESUMO: A Síndrome Pós-Pólio (SPP) caracteriza-se por um novo episódio de fraqueza muscular e/ou fadiga muscular anormal em indivíduos que apresentaram poliomielite anterior aguda (PAA) anos antes. Ainda são poucos os relatos na literatura sobre o gerenciamento da dor na SSP. O objetivo deste trabalho é analisar o controle da dor na síndrome pós-pólio por meio de um relato de caso. Um homem de 57 anos com SPP foi submetido a 24 sessões de fisioterapia para controle do quadro álgico na síndrome, baseada em recursos eletrotermofototerápicos e cinesioterapia. Para análise da dor pré e pós-intervenção fisioterapêutica foram aplicados dois instrumentos: a Escala Analógica Visual (EVA) e a Intensidade de Dor Presente (ID). O quadro álgico no joelho direito sofreu redução de grau 8 para 4 pela EVA e grau 5 para 2 pela ID, enquanto a dor no tornozelo direito reduziu de grau 2 pela EVA e grau 1 pela ID para zero em ambas as escalas. A dor é apontada como um sintoma predominante por muitos pacientes com SPP. O sujeito deste relato, após o programa de tratamento, apresentou melhora expressiva da dor. Entretanto, devido à escassez da literatura sobre dados substanciais referentes ao seu gerenciamento, especialmente na fisioterapia, novos estudos devem ser propostos para gerar maior fundamentação teórica à abordagem fisioterapêutica na SPP.

Descritores: Síndrome Pós-poliomielite; fisioterapia; dor.

ABSTRACT: The Post-polio Syndrome (PPS) is characterized by a new episode of muscular weakness and/or abnormal muscular fatigue in subjects that presented acute previous polio (APP) years ago. There still are few studies about the pain management at PPS. The aim of this study is to analyze pain's management in PPS by a case report. A men with PPS, 57 years, was submitted to 24 sessions of physical therapy for pain's control in PPS, based at electro-thermo physical therapy features and therapeutics exercises. For pain's analysis, pre and post physical therapy interventions were applied two instruments: the Analogic Visual Scale (AVS) and Present Pain Intensity (PPI). The pain in right knee was reduced from grade 8 to 4 on AVS, grade 5 to 2 on PPI, while the pain in his right ankle decreased from grade 2 on AVS and 1 on PPI to zero in both scales. The pain is the predominant symptom for many patients with PPS. This relate, the patient after program of treatment presented an improvement of pain. However, because of there are few studies in the literature about the pain management, especially in physical therapy, new studies must be proposed to generate more theorical at Physical therapy intervention in PSS.

Keywords: Postpoliomyelitis Syndrome; physical therapy; pain. 


\section{INTRODUCÃO}

A Poliomielite Anterior Aguda (PAA) é uma doença endêmica humana causada por um enterovírus de distribuição mundial que se apresenta sob a forma bifásica. Seus sintomas iniciais são quadro de febre, cefaleia e gastrointestinais, seguidos por uma rápida paralisia de caráter assimétrico, decorrente do comprometimento dos neurônios motores da ponta anterior da medula espinhal ${ }^{1,2}$.

Inúmeros pacientes que desenvolveram a doença durante as epidemias das décadas de 1940 e 1950 passaram a apresentar os efeitos tardios da PAA, que incluem um novo quadro de fraqueza muscular associada à fadiga anormal, atrofia e dor, caracterizando uma condição conhecida como Síndrome Pós-Poliomielite (SPP) $)^{1,3,4}$. Tais efeitos estão relacionados aos danos causados pelo vírus na fase aguda e à relativa atividade física durante a fase de estabilidade clínica, provocando a degeneração dos brotamentos axonais das unidades motoras gigantes que se desenvolveram durante a recuperação da $\mathrm{PAA}^{2}$. A incidência e prevalência da SPP são desconhecidas no mundo e no Brasil. Segundo a Organização Mundial da Saúde (OMS), estima-se 12 milhões de pessoas em todo o mundo com algum grau de limitação física causada pela poliomielite $^{5}$.

A dor nos músculos e nas articulações é o primeiro ou segundo sintoma mais comum relatado pelos pacientes, na maioria dos estudos. No que diz respeito à dor muscular, foi referida por 43 a 80\% dos indivíduos com pós-poliomielite no trabalho desenvolvido por Chetwynd et al. ${ }^{6}$. Apesar de a dor constituir um sintoma sine qua non para direcionamento das propostas terapêuticas na SPP, ainda são escassos os relatos na literatura sobre o referido sintoma, tanto no âmbito clínico quanto no campo da fisioterapia. Especialmente pelo número pequeno de casos, já que a doença vem sendo erradicada em nosso país desde 1989 com a ação da vacinação em massa de crianças. Baseado nesta premissa, o presente trabalho visa analisar, por meio de um relato de caso, o efeito de uma estratégia fisioterapêutica no gerenciamento da dor na SSP.
METODOLOGIA

\section{Relato de caso}

O estudo foi realizado no Serviço de Reabilitação Neurológica da Associação Niteroiense de Deficientes Físicos (ANDEF). A concordância em participar da pesquisa foi obtida a assinatura do termo de consentimento livre e esclarecido (TCLE), conforme preconizado pela Resolução 196/96 do Conselho Nacional de Saúde e aprovado pelo Comitê de Ética da instituição, sob o número 043/2009.

PCG, masculino, 57 anos, professor, relata que no primeiro ano de vida apresentou quadro de PAA que levou ao comprometimento do membro inferior direito, gerando deficiência física com limitação relacionada principalmente à deambulação. Após quatro décadas de estabilidade clínica, deu-se início um novo evento de perda funcional, principalmente com dificuldades em subir e descer escadas e executar longas caminhadas. Em maio de 2009, recebeu o diagnóstico de SPP. O exame clínico apresentou encurtamento de $17,3 \mathrm{~cm}$ no membro inferior direito, deformidade em equinocavo-varo com bloqueio da articulação tibiotársica, genu recurvatum, genu valgo e paresia dos músculos proximais e intermediários, com paralisia dos grupamentos distais. Locomovia-se com uso de muleta canadense, com apoio do membro superior esquerdo, sem compensação ortopédica como palmilha ou outro recurso ortótico para diferença entre os membros. Realizava, o apoio no solo com os dedos do membro inferior direito em hiperextensão. Não havia evidência de derrame articular significativo nas articulações do membro inferior direito. Relatava intenso quadro álgico espontâneo na articulação do joelho tíbio-társica direito. As escalas utilizadas para quantificar a dor foram Visual Analógica (EVA) e a Intensidade da sua Dor Presente (ID), antes e após as sessões de fisioterapia ${ }^{7,8}$. A EVA é muito utilizada e consiste em quantificar a dor com uma régua com valores de 0 a 10 sendo que o primeiro representando sem dor e o outro, dor insuportável. A articulação do joelho direito apresentava grau 8 pela EVA e grau 5 pela ID e havia algia mais branda na articulação tíbio-társica direita (grau 2 pela EVA, grau 1 pela ID). A sintomatologia dolorosa intensificava durante palpação articular das respectivas regiões, principalmente quando em posição ortostática e durante a deambulação.

\section{Procedimento}

O sujeito foi submetido a uma avaliação neurológica e ortopédica com duração aproximada de 60 minutos. Enfatizou-se o exame de força muscular, a análise dos reflexos, análise cinemática do membro inferior e a análise da intensidade e reatividade da dor. A análise cinemáticofuncional foi conduzida de acordo com a proposta de avaliação qualitativa de Kisner \& Colby ${ }^{9}$ no que tange os movimentos osteocinemáticos e artocinemáticos, como também a sensação final de movimento, movimento ativo e passivo de todas as articulações do membro inferior.

Após a coleta de dados iniciais, foi definida a abordagem terapêutica empregada. Foram três meses de tratamento fisioterapêutico, que totalizou 24 atendimentos (frequência de dois semanais com a duração de 50 minutos por sessão). No término deste período, realizou-se uma reavaliação do quadro, com ênfase das variáveis analisadas previamente (força, reflexos, intensidade da dor e análise cinemática do membro inferior).

Após avaliação física, concluiu-se que a sintomatologia dolorosa tinha causa articular proveniente das adaptações pósturofuncionais do membro inferior direito. Sendo assim, optou-se por uma abordagem fisioterapêutica que associava a aplicação de Estimulação Elétrica Transcutânea (TENS), Light Amplification by Stimulated Emission of Radiation (Laser), mobilização articular pelo conceito Maitland, cinesioterapia passiva com técnicas de alongamento e flexibilização muscular dos grandes grupamentos musculares do membro inferior e estabilização articular por meio de bandagens funcionais. A execução do programa terapêutico seguiu rigorosamente esta sequência. Todas as técnicas descritas apresentam efeito justificado para auxiliar na redução do quadro álgico e promover melhores condições nas atividades de vida diária.

A proposta de tratamento iniciou com a aplicação do TENS, modo convencional, com frequência de 120 pulsos por segundo e duração de 150 milissegundos na articulação do quadril direito, e frequência de 90 pulsos por segundo com amplitude de 120 milissegundos para a articulação do joelho, durante 20 minutos em cada região. A intensidade foi modulada em um nível sensorial forte, modificada 
constantemente para a manutenção de uma sensação de formigamento intenso na região aplicada. Esta modalidade terapêutica tem efeito na supressão da dor com base na teoria "das comportas". Em sequência aplicou-se o laser de Arseneto de Gálio, 904 mm, modo de aplicação pontual, em ambas as articulações, com dosagem de $3 \mathrm{~J} / \mathrm{cm}^{2}$ na área em que se localizava a dor - cerca de 5 pontos na região medial tibiotársica do membro inferior direito e 9 pontos na região póstero-medial do joelho direito. Ao Laser atribui-se a possibilidade de controlar o processo inflamatório e a dor, além de favorecer o processo de cicatrização e melhorar vascularização tecidual por estimular a neovascularização. O estímulo das mitocôndrias celulares gera efeito bioenergético de aumento na produção de ATP intracelular; favorece a produção de ácido araquidônico e a transformação de prostaglandina em prostaciclina, justificando sua ação na melhora do edema e redução da inflamação; promove aumento da endorfina circulante, proporcionando o efeito analgésico na dor inflamatória; e estimula a regeneração celular ${ }^{10}$.

Em seguida, executou-se uma série específica de mobilização e contenção articular em joelho e tornozelo do membro inferior direito. Estas técnicas (mobilização articular, estiramento muscular mantido e bandagem funcional) tinham o intuito de favorecer um alinhamento segmentar mais fisiológico, visto que o quadro álgico em joelho e tornozelo direito poderia estar ligado ao uso funcional do seguimento com as deformidades articulares presentes. Desta forma, estas manobras buscavam promover um arranjo articular mais próximo da harmonia, isto é, contrário ao padrão deformante.

Como abordagem manual realizada nas deformidades genu recurvatum e genu valgo, iniciamos com mobilização da articulação patelo-femural com ênfase nas direções crânio-caudal e látero-medial da patela. A articulação fêmuro-tibial direita foi decoaptada por meio de uma tração axial associada a movimentos de circundução com o joelho em semiflexão. Esta manobra favorece uma melhor organização das estruturas intrarticulares, sendo realizada com o terapeuta segurando o pé do paciente entre suas pernas e inclinando seu corpo levemente para trás para promover a tração axial, enquanto ambas as mãos estão em torno da tíbia, na altura da tuberosidade anterior da tíbia, realizando movimentos de circundução. Tais manobras foram aplicadas durante aproximadamente cinco minutos cada 9 .

As cadeias musculares anterior e lateral de quadril e coxa do membro inferior direito foram alongadas passivamente com estiramentos mantidos por aproximadamente 20 segundos em 3 séries com intervalos de 20 segundos. Os músculos alongados foram: glúteo médio, tensor da fáscia lata, iliopsoas, sartório e quadríceps. Após esta sequência de mobilizações e exercícios, foi realizada a estabilização articular com a aplicação de esparadrapos, técnica denominada bandagem funcional. No joelho, foi realizada a técnica de contenção para genu valgo, colocando estabilizadores em "xis" na face medial do joelho, cruzando por sobre o ligamento colateral medial. Como as deformidades presentes no tornozelo e pé direito se encontravam fixadas, realizamos apenas o deslizamento posterior do talus em relação à tíbia, devido à posição equina do pé, e a mobilização do calcâneo em direção lateral decorrente ao varismo apresentado. A estabilização articular foi feita seguindo a técnica de correção para retropé varo, com tiras de esparadrapo colocadas em " $U$ ", fixadas inicialmente no terço inferomedial da tíbia, passando inferiormente ao calcâneo, e fixadas na face lateral do terço inferior da perna. $\mathrm{O}$ componente cavo foi mantido por opção. Esta estabilização provoca tração na base do calcâneo e estimula a referida estrutura a assumir posição mais fisiológica ${ }^{11}$.
O paciente do presente estudo ainda recebeu orientações para controle da dor e conservação de energia, dentre elas: manutenção do peso, o repouso e a correta utilização de equipamentos de assistência para deambulação.

\section{RESULTADOS}

No início, o paciente apresentava dor no joelho direito grau 8 pela EVA e 5 pela ID, respectivamente traduzidas por intensa e insuportável. Na articulação tibiotársica direita, a dor percebida foi graduada como 2 pela EVA e 1 pela ID, respectivamente definidas como leve e fraca. Após 12 sessões, a dor no joelho tinha reduzido de 8 para 5 pela EVA e de 5 para 3 pela ID, enquanto na articulação tibiotársica direita presenciamos uma diminuição de 2 para 1 pela EVA e de 1 para 0 pela ID.

Tendo como base os índices obtidos sobre a percepção da dor inicialmente, ao final das 24 sessões, que totalizaram 90 dias, contabilizou-se redução inicial de $50 \%$ na dor do joelho direito pela EVA (de 8 para 4 ) nos primeiros 15 dias (4 sessões), enquanto pela ID constatouse decréscimo de $60 \%$ (5 para 2). A dor percebida na articulação tibiotársica direita foi reduzida em $100 \%$ em ambas as escalas nas 20 sessões. Além disso, houve relato de uma maior facilidade para deambular, pois apesar de apresentar dores, a intensidade e frequência eram indubitavelmente menores. O acompanhamento da dor do paciente frente ao tempo de tratamento está discriminado no Gráfico 1.

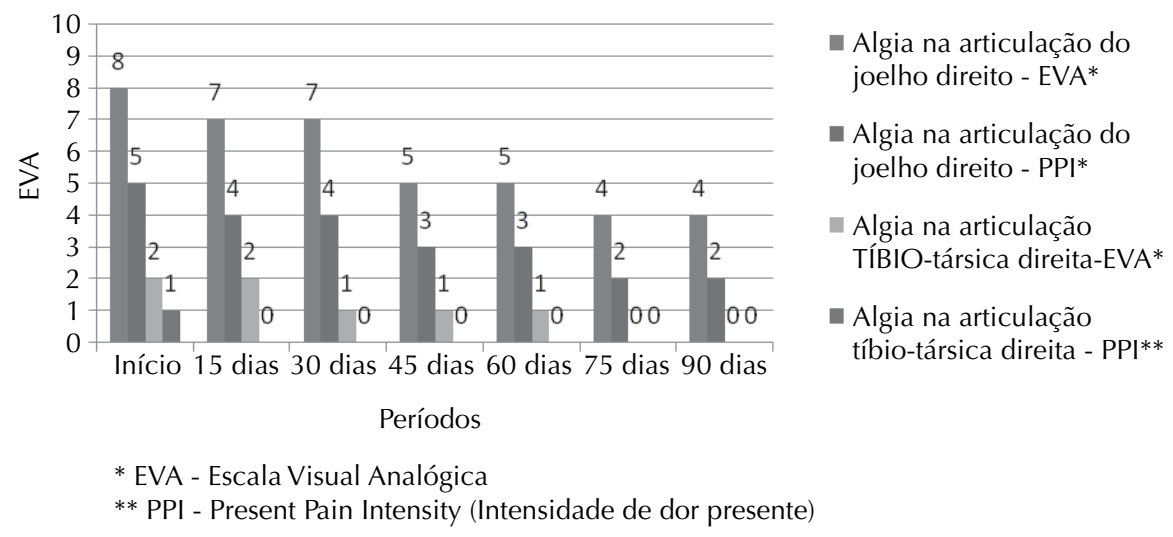

Gráfico 1. Redução da dor com a progressão do tratamento fisioterapêutico 


\section{DISCUSSÃO}

A SPP é uma doença lentamente progressiva, com início geralmente insidioso, que pode levar a deficiências e incapacidades, acarretando restrições funcionais nas atividades básicas e instrumentais da vida diária. Caracteriza-se pelo desenvolvimento de novos sintomas neuromusculares, após um mínimo de 15 anos de estabilidade clínica ${ }^{1,12,13}$. O intervalo médio entre a PAA e as primeiras manifestações da SPP é de aproximadamente 35 anos $^{5,14,15}$.

As manifestações clínicas mais frequentemente encontradas são fraqueza muscular, fadiga e dor ${ }^{14,15}$. A dor é apontada como um sintoma predominante por muitos pacientes, como o do presente estudo, podendo ser de origem muscular, articular ou mioarticular ${ }^{5,16}$. É mais frequente nos membros inferiores, e no dorso daqueles capazes de deambular, e nos membros superiores dos que utilizam cadeiras de rodas ou muletas, estando geralmente associada ao uso excessivo da musculatura remanescente ${ }^{17}$. Pacientes com dor podem limitar suas atividades físicas, desenvolvendo fraqueza e atrofia por desuso secundariamente ${ }^{14}$. A intolerância ao frio também é um sintoma comumente relatado ${ }^{5}$.

As mulheres são mais recorrentes em reportar dores musculares e/ou articulares na SPP. Um maior número de unidades motoras recrutadas e fraqueza acentuada das extremidades inferiores podem ser fatores adicionais importantes para determinar a dor nas articulações. Ambas as dores (musculares e articulares) estão associadas à redução na qualidade de vida ${ }^{15}$. Em vista disso, é altamente recomendável que os indivíduos com efeitos tardios da poliomielite, principalmente com dor e câimbras, modifiquem seu nível de atividade física ${ }^{1,13,15,18}$.

Embora a etiologia da SPP seja desconhecida, hipóteses relacionadas a mecanismos autoimunes apontam a presença de bandas oligloconais e linfócitos no líquor e na medula espinhal de alguns pacientes, além da persistência do poliovírus no sistema nervoso central, demonstrado pelo acúmulo de anticorpos do tipo imunoglobulina $M$ (IgM) no líquor de pacientes com SPP6,19. Entretanto, a teoria mais provável faz relação ao uso excessivo das unidades motoras remanescentes ao longo dos anos numa demanda metabólica intensa. O vírus pode danificar até 95\% dos neurônios motores da medula espinhal, ou mesmo $100 \%$ em pelo menos $50 \%$ dos pacientes. Com a lesão desses neurônios, os músculos de sua área de atuação tornam-se desnervados, ocasionando paresia e atrofia. Embora danificados, os neurônios remanescentes compensam o dano enviando ramificações para ativar os músculos antes desnervados. Em vista disso, a função neuromuscular é recuperada, parcial ou totalmente, dependendo do número de neurônios envolvidos no processo. Um único neurônio pode lançar derivações para conectar cinco a dez vezes mais fibras musculares do que fazia originalmente. O grande problema dá-se quando tal população de neurônios remanescentes é sobrecarregada por muitos anos. Inicia-se um novo processo degenerativo com surgimento de um novo quadro sintomatológico ${ }^{20}$.

Os possíveis fatores de risco para o desenvolvimento da SPP estão ligados à gravidade da PAA, sexo feminino, idade avançada na época do episódio, deficiências permanentes após a recuperação, ganho recente de peso e maior atividade física durante o período de estabilidade ${ }^{21-24}$. O indivíduo apresentado em nosso estudo realizou intensa atividade física durante a fase de estabilidade clínica, fator apontado como um dos principais agentes etiológicos envolvidos na gênese da SPP.

Os principais critérios para se estabelecer o diagnóstico de SPP são: história confirmada de PAA, período de recuperação parcial ou completa, seguido de um período de estabilidade clínica de pelo menos 15 anos, início gradual, ou raramente abrupto, de nova fraqueza muscular ou fadiga anormal, e a exclusão de outras condições neurológicas ou ortopédicas ${ }^{18,21}$. Vale ressaltar que o paciente estudado apresenta os critérios supracitados. Sabe-se que o controle da dor pode ser realizado por uso de calor, frio, eletroterapia e medicamentos, entretanto as atividades de alongamento e "fortalecimento" são fundamentais por atuarem de maneira direta na articulação e no músculo ${ }^{18,21}$.

Mesmo não sendo o foco deste estudo, os exercícios de fortalecimento muscular têm contribuído para o gerenciamento da dor. Estudos não controlados envolvendo exercícios isotônicos, isocinéticos e isométricos mostraram-se eficazes na redução da fadiga, da fraqueza muscular e da dor em pacientes portadores da SPP. Programas terapêuticos controlados, incluindo treino ergométrico em membros superiores e inferiores, condicionamento de marcha, exercícios aeróbicos e atividades aquáticas, também forneceram resultados significativos nessa mesma população ${ }^{22,23}$. Os indivíduos que padecem de síndrome pós-polio devem ser submetidos a atividades terapêuticas (cinesioterapia) em limites submáximos. A proposta terapêutica descrita neste relato de caso tem como ponto norteador as atividades funcionais e o controle da dor. Sendo assim, justifica-se a utilização de recursos pré-cinesioterapêuticos, como o TENS e o Laser, para o gerenciamento da dor e a permissão de que haja melhor resposta funcional do membro acometido pela pólio. Uma grande vantagem da aplicação do TENS e Laser é o efeito analgésico e de controle inflamatório e a restrição do uso da crioterapia, já que cerca de $70 \%$ dos pacientes apresentam intolerância ao frio ${ }^{6}$. No que tange a prática de exercícios ativos livres, entram em consonância com as recomendações de diversos estudos ${ }^{2,4}$ sobre apenas a necessidade de recrutamento muscular sem gerar fadiga. Portanto, eles e as técnicas de alongamento têm a capacidade minimizar os danos do desuso sem gerar sobrecarga ${ }^{9}$. Referente à utilização da bandagem, neste relato de caso o recurso tem o intuito de promover a funcionalidade e um padrão de posicionamento articular contra as mudanças estruturais articulares desencadeadas pela pólio ${ }^{9}$. Com a sua otimização pela técnica de Maitland, que objetiva a melhorar da artrocinemática e minimizar o quadro álgico articular $^{9}$, a proposta de nosso estudo pode promover resultados satisfatórios no controle da dor.

As deficiências causadas pelo surgimento dos novos sintomas, somadas às deficiências primárias provocadas pela PAA, provocam grandes restrições. As intervenções terapêuticas na SPP têm como objetivo fornecer aos pacientes princípios e métodos para o autocuidado, efetuando mudanças no estilo de vida e reduzindo a carga metabólica excessiva sobre os neurônios motores. As condutas terapêuticas adotadas devem preconizar a utilização de técnicas de conservação de 
energia, orientações sobre períodos regulares de repouso, perda de peso e prática de exercícios em níveis que evitem o uso excessivo, além de indicação para a utilização de órteses, quando necessário ${ }^{18}$. O ponto-chave do tratamento é prevenir a deterioração subsequente por meio do equilíbrio entre a atividade e o repouso.

Stoelb et al. ${ }^{24}$, buscando descrever a frequência, a intensidade e o impacto da dor em indivíduos vitimados pela SPP, realizaram um estudo retrospectivo e crosssectional com 63 pacientes com dores em diversas regiões do corpo. Os locais de maior frequência foram os ombros, a coluna lombar, as pernas e os quadris. Com relação à intensidade da dor, joelho, pernas, punhos, coluna lombar e a cabeça (cefaleia) foram às áreas mais frequentemente comprometidas. A presença de dor contribuiu negativamente na qualidade do sono e na execução de atividades da vida diária que exigiam um intenso trabalho muscular.

Conde et al. ${ }^{20}$ tiveram o intuito de descrever os aspectos clínicos e epidemiológicos de pacientes com SPP, além de identificar os fatores preditivos para a gravidade da evolução. Assim, realizaram um estudo com 132 pacientes no Setor de Doenças Neuromusculares da Universidade Federal de São Paulo. A média de início dos primeiros sintomas foi de 39,4 anos. Os mais comuns apresentados pela amostra foram: a fadiga $(87,1 \%)$, as dores musculares $(82,4 \%) \mathrm{e}$ as dores articulares $(72,0 \%)$. Os pesquisadores concluíram que os profissionais envolvidos diretamente no atendimento dessa clientela devem ser minuciosos na avaliação inicial e no acompanhamento, além de atentarem para as implicações negativas do "supertreinamento" durante o período de recuperação.

Segundo as diretrizes da Sociedade Europeia de Neurologia ${ }^{21}$, fundamentadas a partir de pesquisas aos principais periódicos de expressão científica, os pacientes com SPP necessitam de intensa supervisão. Com relação à dor, os pesquisadores alertaram que o treinamento muscular inadequado pode exacerbá-la e contribuir para episódios agudos (Nível B). Perda de peso, utilização de equipamentos de assistência e suporte devem ser considerados devido a satisfatórios resultados práticos obtidos. Inúmeros tratamentos para SPP foram concluídos. Entretanto, no que tange ao gerenciamento da dor, nenhum efeito terapêutico definitivo tem sido relatado para os agentes farmacológicos utilizados, como piridostigmina, corticóides e amantadine. Novos estudos randomizados (clínicos e de reabilitação) devem direcionar-se para o tratamento da dor, um sintoma comumente relatado pelos pacientes com SPP. Em adição, é necessário que haja pesquisas de longo prazo para caracterização dos efeitos em um tempo mais prolongado do treinamento muscular.

\section{CONCLUSÃO}

Os resultados deste relato de caso apontam para uma possibilidade real no gerenciamento da dor por parte da intervenção fisioterapêutica. Nossos dados sugerem melhorias sobre as sequelas tardias da poliomielite. Inúmeros tratamentos clínicos têm sido testados e utilizados para o gerenciamento da dor em pacientes com SPP. Entretanto, a adesão e um correto esclarecimento sobre o tipo, frequência e intensidade dos programas ainda são escassos na prática clínica e na literatura vigente, principalmente no âmbito da abordagem fisioterapêutica. Embora o presente trabalho apresente limitações de um relato de caso, tais reflexões são de grande importância para a formulação de novos estudos, com um tamanho amostral expressivo, preferencialmente ensaios clínicos randomizados-controlados. Assim, poderemos elucidar quais são as estratégias mais eficazes para gerenciamento da dor na SPP.

\section{REFERÊNCIAS}

1. Sunnerhagen KS, Grimby G. Muscular effects in late polio. Acta Physiol Scand. 2001;171(3):335-40.

2. Howard RS. Poliomyelitis and the postpolio syndrome. BMJ. 2005;330(7503):1314-8.

3. Rekand T, Albrektsen G, Langeland N, Aarli JA. Risk of symptoms related to late effects of poliomyelitis. Acta Neurol Scand. 2000;101(3):153-8.

4. Rekand T, Kõrv J, Farbu E, Roose M, Gilhus NE, Langeland $\mathrm{N}$, et al. Long term outcome after poliomyelitis in different health and social conditions. J Epidemiol Community Health. 2003;57(5):368-72.

5. Secretaria de Estado da Saúde de São Paulo. Divisão de Doenças de Transmissão Hídricas e Alimentares. Centro de Vigilância Epidemiológica "Alexandre Vranjac". Coordenadoria de Controle de Doenças. Síndrome póspoliomielite. Rev. Saúde Pública. 2006;40(5):941-5.

6. Chetwynd J, Botting C and Hogan D. Postpolio syndrome in New Zealand: a survey of 700 polio survivors. N Z Med J. 1993;106(964):406-8.
7. Melzack R, Katz J. Measurement of pain. Surg Clin North Am. 1996;79(2):231-52.

8. Wells-Federman C, Arnstein P, Caudill M. Nurse-led pain management program: effect on self-efficacy, pain intensity, pain-related disability, and depressive symptoms in chronic pain patients. Pain Manag Nurs. 2002;3(4):131-40.

9. Kisner C, Colby LA. Exercícios terapêuticos: fundamentos e técnicas. $4^{a}$ ed. São Paulo: Manole; 2005.

10. Marovino T. Cold lasers in pain management. Practical Pain Management. 2004;4(6):37-42.

11. Austin K, Gwynn-Brett, Marshall S. Illustrated guide to taping techniques. London, UK: Ed Wolfe; 1994.

12. Leparc-Goffart I, Julien J, Fuchs F, Janatova I, Aymard $\mathrm{M}$, Kopecka $\mathrm{H}$. Evidence of presence of poliovirus genomic sequences in cerebrospinal fluid from patients with postpolio syndrome. J Clin Microbiol. 1996;34(8):2023-6. 


\section{Referências (cont.)}

13. Halstead LS, Rossi CD. Post-polio syndrome: clinical experience with 132 consecutive outpatients. Birth Defects Orig Artic Ser. 1987;23(4):13-26.

14. Dalakas M, Illa I. Post-polio syndrome: concepts in clinical diagnosis, pathogenesis, and etiology. Adv Neurol. 1991;56:495-511.

15. Trojan DA, Cashman NR. Post-poliomyelitis syndrome. Muscle Nerve. 2005;31(1):6-19.

16. Koopman FS, Uegaki K, Gilhus NE, Beelen A, de Visser M, Nollet F. Treatment for postpolio syndrome. Cochrane Database Syst Rev. 2011;16(2):CD007818.

17. Grimby G, Stalberg E, Sandberg A, Sunnerhagen KS. An 8-year longitudinal study of muscle strength, muscle fiber size, and dynamic electromyogram in individuals with late polio. Muscle Nerve. 1998;21(11):1428-37.

18. Orsini M, Mello MP, Antonioli RS, Nascimento OJM, de Freitas MRG. . Post-Polio Syndrome and the motor rehabilitation process: case report. A síndrome pós-polio e o processo de reabilitação motora: relato de caso. Rev Neurocienc. 2008;15:321-5.
19. Jubelt B, Cashman NR. Neurological manifestations of the post-polio syndrome. Crit Rev Neurobiol. 1987;3(3):199-220.

20. Conde MT, Oliveira AS, Quadros AA, Moreira GA, Silva HC, Pereira RD et al. Post-polio syndrome: epidemiologic and prognostic aspects in Brazil. Acta Neurol Scand. 2009;120(3):191-7.

21. Farbu E, Gilhus NE, Barnes MP, Borg K, de Visser M, Driessen A, et al. EFNS guideline on diagnosis and management of post-polio syndrome. Report of an EFNS task force. Eur J Neurol. 2006;13(8):795-801.

22. Jubelt B. Post-polio syndrome. Curr Treat Options Neurol. 2004;6(2):87-93.

23. On AY, Oncu J, Atamaz F, Durmaz B. Impact of postpolio-related fatigue on quality of life. J Rehabil Med. 2006;38(5):329-32.

24. Stoelb BL, Carter GT, Abresch RT, Purekal S, McDonald $C M$, Jensen MP. Pain in persons with postpolio syndrome: frequency, intensity, and impact. Arch Phys Med Rehabil. 2008;89(10):1933-40. 